\title{
Influence of Radiative Heat Energy on the MHD Flow of Cu-kerosene Nanofluid Over a Vertical Plate: Laplace Transform Technique
}

\author{
R.K. Dash ${ }^{1(\mathbb{D})}$, S.R. Mishra ${ }^{1}$ (D), P.K. Pattnaik ${ }^{2, *}$ (D) \\ 1 Department of Mathematics, SikshaO' Anusandhan Deemed to be University, Bhubaneswar, Odisha, 751030, India; \\ rishikanta.dash5@gmail.com (R.K.D.); satyaranjan_mshr@yahoo.co.in (S.R.M.); \\ 2 Department of Mathematics, College of Engineering and Technology, Bhubaneswar, Odisha, 751029, India; \\ papun.pattnaik@gmail.com (P.K.P.); \\ * Correspondence: papun.pattnaik@gmail.com (P.K.P.);
}

Scopus Author ID 56659255500

Received: 3.07.2021; Revised: 25.09.2021; Accepted: 29.09.2021; Published: 8.11.2021

\begin{abstract}
The present study reveals the heat transfer phenomena of magnetohydrodynamic (MHD) nanofluid over a moving vertical plate due to the interaction of thermal radiation. Due to enhancing thermal properties, instead of water, kerosene is treated as the base fluid with the inclusion of $\mathrm{Cu}$ (Copper) nanoparticles. In addition to that, for the nanofluid viscosity and thermal conductivity the Einstein's model and Mintsa's model, respectively. The transformed models for the governing equations proposed here are handled analytically employing Laplace Transform Technique. However, the variations of various parameters are obtained because the constructed flow phenomena are presented via graph. In the particular case, the consequence obtained is compared with the earlier study to get the validation which provides a road map for further investigation.
\end{abstract}

Keywords: MHD; Cu-kerosene nanofluid; thermal radiation; Laplace Transform Technique.

(C) 2021 by the authors. This article is an open-access article distributed under the terms and conditions of the Creative Commons Attribution (CC BY) license (https://creativecommons.org/licenses/by/4.0/).

\section{Introduction}

The development in the field of heat transport phenomena is one of the important aspects of various researchers in the recent era. Due to the varied application in industries and engineering for the production of electronic devices and manufacturing processes, the shape and size of the product depend upon the cooling of the system. In earlier studies [1], it is seen that the use of conventional fluid is less effective for the same process; therefore, the young ignited mind develop their new research by using the mixture of nanoparticles with the conventional liquid. The fact is that the higher conductivity of the nano-sized particles overrides the conductivity of the conventional fluid, which is useful for enhancing the heat transport phenomena. The metals and oxides and carbides embedding with the water, kerosene, and most probably the engine oil, ethyl glycol, etc., are proposed as nanofluids that are useful for the transport of heat. However, carbon nanotubes having single-walled or multi-walled are also beneficial for the enrichment of the transport criteria.

Several industries can code the chemical reaction as non-uniform or uniform procedures due to the manufacturing processes. Gurivi et al. [1] inspected the impact of the reaction of chemical, thermal diffusion, and thermal radiation on unstable public transport by the simultaneous flow of convective mass and energy transport of condensed electrically 
conductive liquid over a semi-infinite rotating permeable sheet surrounded by a permeable media. Flowing liquids through the porous medium is of the utmost importance as they are quite common in nature. Such motions have caught the consideration of scholars and inventors because of their varied assortment of applications in several fields of discipline and engineering.

The chemical reaction processes depend on whether they are on the boundary or as a single-phase volume response. In the process of thorough mixing, when the interface energy level occurs, the reaction is heterogeneous; when it occurs in solution, the reaction is homogeneous. In the process of the reactive agent, the rate depends on the quality of the substance. When the reaction rate is proportional to the mass itself, it is called a first-order reaction. In numerous chemical manufacturing procedures, a reaction of chemicals arises among the external matter and the liquid to which the sheet is revolving. These procedures occur in many manufacturing applications. The first-order chemical reaction near a horizontal sheet was investigated by Chambre and Young [2]. Chen and Yuh [3] considered the effect of mixing energy and material under wall heat/mass and uniform heat / mass flux conditions. Patil and Kulkarni [4] have examined the control of the chemical reactions and radiative heat on the free convection current under the impact of variable suction force. Magnetohydrodynamic momentary of free convection and chemically volatile motion over a permeable vertical plate has been surveyed by Tripathy et al. [5]. Rout et al. [6] have analyzed the natural convection current of the magnetohydrodynamic laminar flow in a micropolar liquid with a chemical reaction with a transverse magnetic description, variable mass, and wall energy over a vertical plate. Jena et al. [7] investigated the impact of various flow phenomena in Jeffrey fluid flow along with a stretchable plate in conjunction with the magnetic field. They observed that elasticity, magnetic fields, and porous media prevented Jeffery liquids from moving as a porous medium, and they also noticed that magnetic fields helped enhance the energy field in the motion field. The oscillating blood flow of MHD in porous arterioles with an outward magnetic description is conveyed by Misra and Adhikary [8]. Tripathy [9] reported an investigation of an electrically conductive viscous liquid along with a rotating perpendicular sheet over a permeable surface in conjunction with energy and solutal transfer impact with a heat source. Ahmed et al. [10] Demonstrated 3-dimensional channel flow fluid among two long perpendicular plane sheets under the influence of a lateral magnetic description. They pointed out that an increase in permeability or magnetic parameters will augment the speed of the moving board. The increase in Reynolds number increases the x component on the stationary wall and reduces the $\mathrm{z}$ component. The viscous dissipation plays an important character in geophysical flows and convinced manufacturing procedures and is usually considered by the Eckert number. Soundalgekar [11] analyzed viscid dissipative effects on the unstable natural convection current across an infinite perpendicular permeable sheet; once the energy fluctuates with time, there is persistent suction on the surface. Makinde and Mishra [12] scrutinized the heat transfer of a viscid condensed fluid in a permeable medium past an oscillating perpendicular surface. The influence of radiative heat on the motion of magnetohydrodynamics has become very important in industrial applications. Different production processes arise at high energy, and, therefore, data of radiation and temperature transport is vital for the design of engineering apparatus. Nuclear power plants, gas turbines, and numerous aircraft, missile, and satellite propulsion devices are illustrations of such procedures [13]. The influence of radiation on a porous vertical surface has been scrutinized by Hossain et al. [14]. Makinde [15] has reported the mass transfer and natural convection boundary current with thermal radiation 
across a rotating perpendicular permeable sheet. The impact of radiation, Joule heat, and dissipative heat on the laminar MHD flow past a smooth sheet of the Marangoni convection is examined by Rohana et al. [16]. Babu et al. [17] have analyzed the impact of thermal properties on MHD flow along a shrinking surface. Makinde and Mishra [18] have reported the magnetohydrodynamic motion of uninterruptedly rotating perpendicular sheets under the influence of temperature and concentration flux and chemical reaction over a permeable media. The impacts of energy and concentration transport on the free convective current of MHD along with the disposed sheet in a permeable media have been inspected by Mishra et al. [19].

The momentum slips impact the energy and concentration fluxes of magnetohydrodynamic viscid electrically conductive fluid motion together with a stretchable plate with viscid dissipation is analyzed by Kayalvizhi et al. [20]. Rashidi et al. [21] analyzed a semi-analytical solution of 2-D boundary layer viscous flow within a permeable domain of MHD elastico-viscous liquid with heat source/sink. The impact of temperature and concentration flux properties on the magnetohydrodynamic time-independent flow of radiative Jeffery nanofluid with cross-diffusion behavior was examined by Abbasi et al. [22]. The influence of buoyant forces on energy and concentration transport made by a persistent mass flux on a perpendicular sheet with variable heat has been investigated by Geetha and Muthucumaraswamy [23]. They noticed that once the mass Grashof number rises, the skin friction values decline. They also noted that shear stress values decrease over time. Hayat et al. [24] surveyed the impact of Brownian motion and thermophoresis, and thermal radiation on the 3D flow of Powell-Eyring nano liquid due to flux conditions. Some more [25-40] interesting results about MHD and nanofluid flow have been cited to enhance the study.

The present investigation deals with analyzing the time-dependent free convective flow of nanofluid past a vertical surface. Electrically conducting nanofluid presents the impact of radiative heat energy, thermal and solutal buoyancy, and chemical reactions on the flow phenomena. The unsteady flow problem with the set of transformed non-dimensional equations is handled by the Laplace transformation method. The characteristics of the contributing parameters are displayed graphically, and the corroboration of the current investigation is acquired with the earlier work in the case of pure fluid that shows a good agreement.

\section{Mathematical Formulation}

The time-dependent flow of nanofluid over a moving vertical surface is considered due to the interaction of the transverse magnetic field and the impact of buoyant forces. Initially, the plate is at rest, and the plate temperature and concentration are considered to be constant. Along the vertical direction, the plate starts moving with velocity $\lambda u_{0}, t>0$. The wall temperature and concentration are either increased or decreased correspondingly as $T_{w}$ and $C_{w}$

Here, with an assumption of low "Magnetic Reynolds number", it is wise to neglect the impact of induced magnetic field and Hall current. Thus, the proposed flow phenomena are as follows:

$\nabla . u=0$,

$\rho_{n f} \frac{\partial U}{\partial t}=-\nabla p+\mu_{n f} \nabla^{2} U+J \times B+g b$

$\left(\rho C_{p}\right)_{n f} \frac{\partial T}{\partial t}=-\nabla . q$, 
$\frac{\partial C}{\partial t}=-\nabla \cdot j$

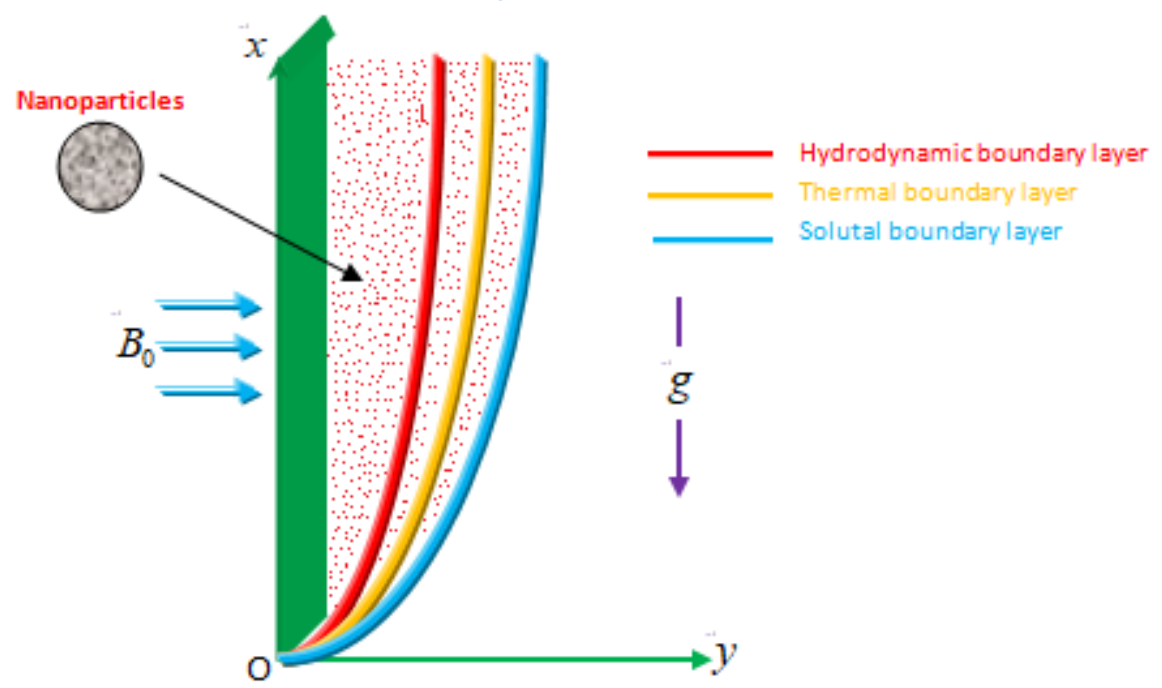

Figure 1. Flow geometry.

Here $U=(u, v, w)$ denotes the velocity vector, and the flow is in an upward direction along the $\mathrm{x}$-axis, and the $\mathrm{y}$-axis is normal to it. The flow is confined to the positive direction of $\mathrm{y}$ as the plane $y=0$, the plate coincides. Let a uniform transverse magnetic field $B_{0}$ and radioactive flux $q_{r}$ are along the normal direction of flow, and assuming no voltage exists whether applies or polarization and we neglect the pressure gradient. The fluid is conducting electrically, and the water-based nanofluid is dipped with a copper nanoparticle. Now considering the reactive species within the nanofluid and the concentration phenomenon is taken place in the stream.

However, following Das and Jana [41] the governing equations can be expressed as;

$\rho_{n f} \frac{\partial u}{\partial t}=\mu_{n f} \frac{\partial^{2} u}{\partial y^{2}}-\sigma_{n f} B_{0}^{2} u+g\left(\rho \beta_{T}\right)_{n f}\left(T-T_{\infty}\right)+g\left(\rho \beta_{C}\right)_{n f}\left(C-C_{\infty}\right)$,

$\left(\rho c_{p}\right)_{n f} \frac{\partial T}{\partial t}=k_{n f} \frac{\partial^{2} T}{\partial y^{2}}-\frac{\partial q_{r}}{\partial y}+Q_{0}\left(T-T_{\infty}\right)$

$\frac{\partial C}{\partial t}=D \frac{\partial^{2} C}{\partial y^{2}}-K_{1}\left(C-C_{\infty}\right)$.

The appropriate boundary conditions are:

$t=0: \quad u=0, T=T_{\infty}, C=C_{\infty} \quad \forall y \geq 0$,
$t>0:\left\{\begin{array}{lc}u=\lambda u_{0}, T=T_{w}, C=C_{w}, & \text { at } y=0, \\ u \rightarrow 0, T \rightarrow T_{\infty}, C \rightarrow C_{\infty} & \text { as } y \rightarrow \infty\end{array}\right\}$.

The effective properties like viscosity (Einstein model [42]), conductivity (Mintsa model [43]), and other properties are tabulated as $\left.\begin{array}{l}\mu_{n f}=(1+2.5 \phi) \mu_{f},\left(\rho \beta_{T}\right)_{n f}=(1-\phi)\left(\rho \beta_{T}\right)_{f}+\phi\left(\rho \beta_{T}\right)_{s},\left(\rho c_{p}\right)_{n f}=(1-\phi)\left(\rho c_{p}\right)_{f}+\phi\left(\rho c_{p}\right)_{s}, \\ \left(\rho \beta_{C}\right)_{n f}=(1-\phi)\left(\rho \beta_{C}\right)_{f}+\phi\left(\rho \beta_{C}\right)_{s}, k_{n f}=(1+1.72 \phi) k_{f}, \sigma_{n f}=\sigma_{f}\left(1+\frac{3(\sigma-1) \phi}{\sigma+2-(\sigma-1) \phi}\right), \sigma=\frac{\sigma_{s}}{\sigma_{f}} .\end{array}\right\}$

As earlier, it is defined as the plate velocity is $\lambda u_{0}$, where $\lambda$ stands for the direction of the movement. In particular, $\lambda=0$ it corresponds to the situation that the plate is static and 
$\lambda \neq 0$ represents to moving plate. Now, the consideration of radiative heat flux $q_{r}$ is employed assuming the Rosseland approximation and expressed as (Sheikholeslami and Ganji [44]);

$q_{r}=-\frac{4 \sigma^{*}}{3 k^{*}} \frac{\partial T^{4}}{\partial y}$

where $\sigma^{*} \approx 1.3806 \times 10^{-23}$, the Stefan Boltzmann constant and $k^{*}$, Rosseland's mean absorption coefficient. Further, the expansion of $T^{4}$ about $T_{\infty}$ leads to $T^{4}=T_{\infty}^{4}+3 T_{\infty}^{3}\left(T-T_{\infty}\right)+6 T_{\infty}^{2}\left(T-T_{\infty}\right)^{2}+\ldots$

Considering only the linear terms, the above expression can be written as $T^{4} \approx 4 T_{\infty}^{3} T-3 T_{\infty}^{4}$

Impose of Eq. (11) in Eq. (10) and further using in Eq. (6) we get

$\left(\rho c_{p}\right)_{n f} \frac{\partial T}{\partial t}=k_{n f} \frac{\partial^{2} T}{\partial y^{2}}+\frac{16 \sigma^{*} T_{\infty}^{3}}{3 k^{*}} \frac{\partial^{2} T}{\partial y^{2}}+Q_{0}\left(T-T_{\infty}\right)$

The non-dimensional quantities that appeared in the process of formulating the model are expressed as,

$$
\left.\begin{array}{l}
\eta=\frac{u_{0}}{v_{f}} y, \tau=\frac{u_{0}^{2}}{v_{f}} t, f=\frac{u}{u_{0}}, \theta=\frac{T-T_{\infty}}{T_{w}-T_{\infty}}, \psi=\frac{C-C_{\infty}}{C_{w}-C_{\infty}}, M^{2}=\frac{\sigma_{f} B_{0}^{2} v_{f}}{u_{0} \rho_{f}}, G r=\frac{g \beta_{T f}\left(T_{w}-T_{\infty}\right) v_{f}}{u_{0}^{3}}, \\
G c=\frac{g\left(\beta_{C}\right)_{f}\left(C_{w}-C_{\infty}\right) v_{f}}{u_{0}^{3}}, \operatorname{Pr}=\frac{\left(\mu c_{p}\right)_{f}}{k_{f}}, N r=\frac{4 \sigma^{*} T_{\infty}^{3}}{k^{*} k_{f}}, S=\frac{Q_{0} v_{f}}{u_{0}^{2}\left(\rho c_{p}\right)_{f}}, L e=\frac{\alpha_{f}}{D_{B}}, K c=\frac{K_{1} v_{f}}{u_{0}^{2}} .
\end{array}\right\}
$$

Putting these dimensionless variables in the governing equation becomes

$$
\begin{aligned}
& \frac{\partial f(\tau, \eta)}{\partial \tau}=A_{1} \frac{\partial^{2} f(\tau, \eta)}{\partial \eta^{2}}-A_{2} M f(\tau, \eta)+G r A_{3} \theta(\tau, \eta)+G c A_{4} \psi(\tau, \eta), \\
& \frac{\partial \theta(\tau, \eta)}{\partial \tau}=\frac{1}{\operatorname{Pr}} A_{5} \frac{\partial^{2} \theta(\tau, \eta)}{\partial \eta^{2}}+A_{6} \theta(\tau, \eta),
\end{aligned}
$$$$
\frac{\partial \psi(\tau, \eta)}{\partial \tau}=\frac{1}{L e \operatorname{Pr}} \frac{\partial^{2} \psi(\tau, \eta)}{\partial \eta^{2}}-K c \psi(\tau, \eta),
$$

$$
\begin{gathered}
\text { subject to } \\
\tau=0: \quad f=0, \theta=0, \varphi=0 \\
\tau>0:\left\{\begin{array}{ll}
f=\lambda, \theta=1, \varphi=1 & \forall \eta \geq 0, \\
f \rightarrow 0, \theta \rightarrow 0, \varphi \rightarrow 0 & \text { at } \eta=0,
\end{array}\right\}
\end{gathered}
$$

$a_{1}=1-\phi+\phi \frac{\rho_{s}}{\rho_{f}}, a_{2}=1-\phi+\phi \frac{\left(\rho \beta_{T}\right)_{s}}{\left(\rho \beta_{T}\right)_{f}}, a_{3}=1-\phi+\phi \frac{\left(\rho \beta_{c}\right)_{s}}{\left(\rho \beta_{c}\right)_{f}}, A_{1}=\frac{1}{a_{1}}(1+2.5 \phi)$,

$$
\left.A_{2}=\frac{1}{a_{1}}\left(1+\frac{3(\sigma-1) \phi}{\sigma+2-(\sigma-1) \phi}\right), A_{3}=\frac{a_{2}}{a_{1}}, A_{4}=\frac{a_{3}}{a_{1}}, A_{5}=\frac{1}{a_{4}}\left(\frac{k_{n f}}{k_{f}}+\frac{4}{3} N r\right), A_{6}=\frac{1}{a_{4}} S\right\} \text {. }
$$

The rate coefficients at the surface are described as $S k_{f}$ (skin friction), Nusselt number $(\mathrm{Nu}$ ), and $\mathrm{Sh}$ (Sherwood number).

$$
S k_{f}=-(1+2.5 \phi)\left[\frac{\partial f(\tau, \eta)}{\partial \tau}\right]_{\eta=0}, N u=-\left((1+1.72 \phi)+\frac{4}{3} N r\right)\left[\frac{\partial \theta(\tau, \eta)}{\partial \tau}\right]_{\eta=0}, S h=-\left[\frac{\partial \psi(\tau, \eta)}{\partial \tau}\right]_{\eta=0} .
$$


Table 1. Physical characteristics of kerosene and Copper

\begin{tabular}{c|c|c} 
& Kerosene & $\mathbf{C u}$ \\
\hline$\rho$ & 783 & 8933 \\
\hline$c_{p}$ & 2090 & 385 \\
\hline$k$ & 0.145 & 401 \\
\hline$\beta_{T}$ & $21 \times 10^{-5}$ & $1.67 \times 10^{-5}$ \\
\hline$\beta_{C}$ & $298.2 \times 10^{-6}$ & $3.05 \times 10^{-6}$ \\
\hline$\sigma$ & 0.149 & $59.6 \times 10^{6}$
\end{tabular}

\section{Results and Discussion}

An analysis is performed for the natural convection of kerosene-based nanofluid, including $\mathrm{Cu}$-nanoparticle, past a moving vertical plate. An electrically conducting fluid in conjunction with radiative heat, heat source, and the combination of first-order chemical reactions enhance the study. The crux of the present analysis is the appearance of Einstein's model [42] viscosity and the thermal conductivity model proposed by Mintsa [43]. An analytical approach such as the Laplace transformation technique is employed to solve timedependent problems formulated using the aforesaid assumptions. Table 1 represents the thermophysical properties such as viscosity, conductivity, density, volumetric expansion of both the particle and the base fluid kerosene. The flow phenomena are governed by the assumption of several pertinent parameters, and their characteristic is displayed via Figures 215, and numerical simulation for the rate coefficients is presented in Table 2. Conformity of the convergence criteria, along with an excellent validation of the present results compared to an earlier study of Sheikholeslami and Ganji [44], is obtained in particular cases.

\subsection{Velocity distributions.}

The validation of the velocity profiles along with the characteristics of particle concentration $\phi$ on the velocity distribution is presented in Figure 2. The comparison is obtained for $\phi=0$ with the earlier work of Mahanthesh et al. [45] when magnetic impact $M(M=0)$, heat $\quad$ source $Q(Q=0), \quad$ reactive $\quad$ species $K c(K c=0) . \quad$ Further, $M=1 ; \phi=0.1 ; G r=10 ; G c=10 ; P r=23 ; N r=1 ; S=-0.1 ; L e=2 ; t=0.5 ; K c=0.1 ;$ and $\mathrm{a}=0.1$ are considered to be fixed for the simulation process to carry out the study for the investigation of the behavior of the particular parameters that is shown in the corresponding figures. However, Figure 2 signifies strong retardation in the fluid momentum of the $\mathrm{Cu}$-kerosene nanofluid due to the enhanced nanoparticle concentration. The range of concentration is proposed as $\phi \in[0,0.2]$. The fact is, an enhance in concentration, the heavier density of the nanoparticles clogging near the surface opposes the fluid velocity. As a result, the retardation is marked. However, the case of pure fluid renders its maximum magnitude near the surface as well as in the entire domain. A vector field proposed by the magnetic field describes the magnetic influence on electric current and the corresponding materials that experience a force applied normal to its velocity. 


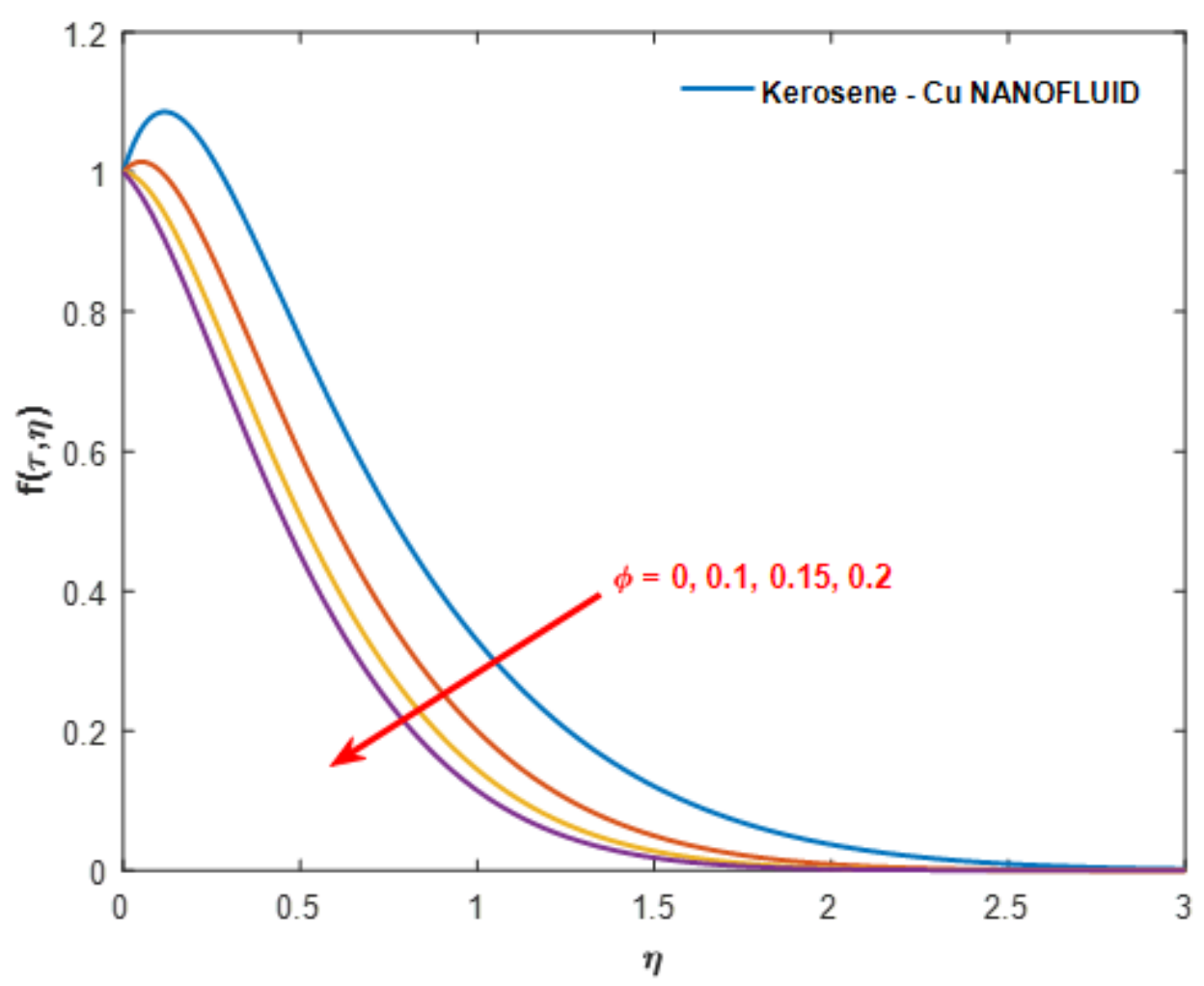

Figure 2. Velocity profile for the impact of $\phi$.

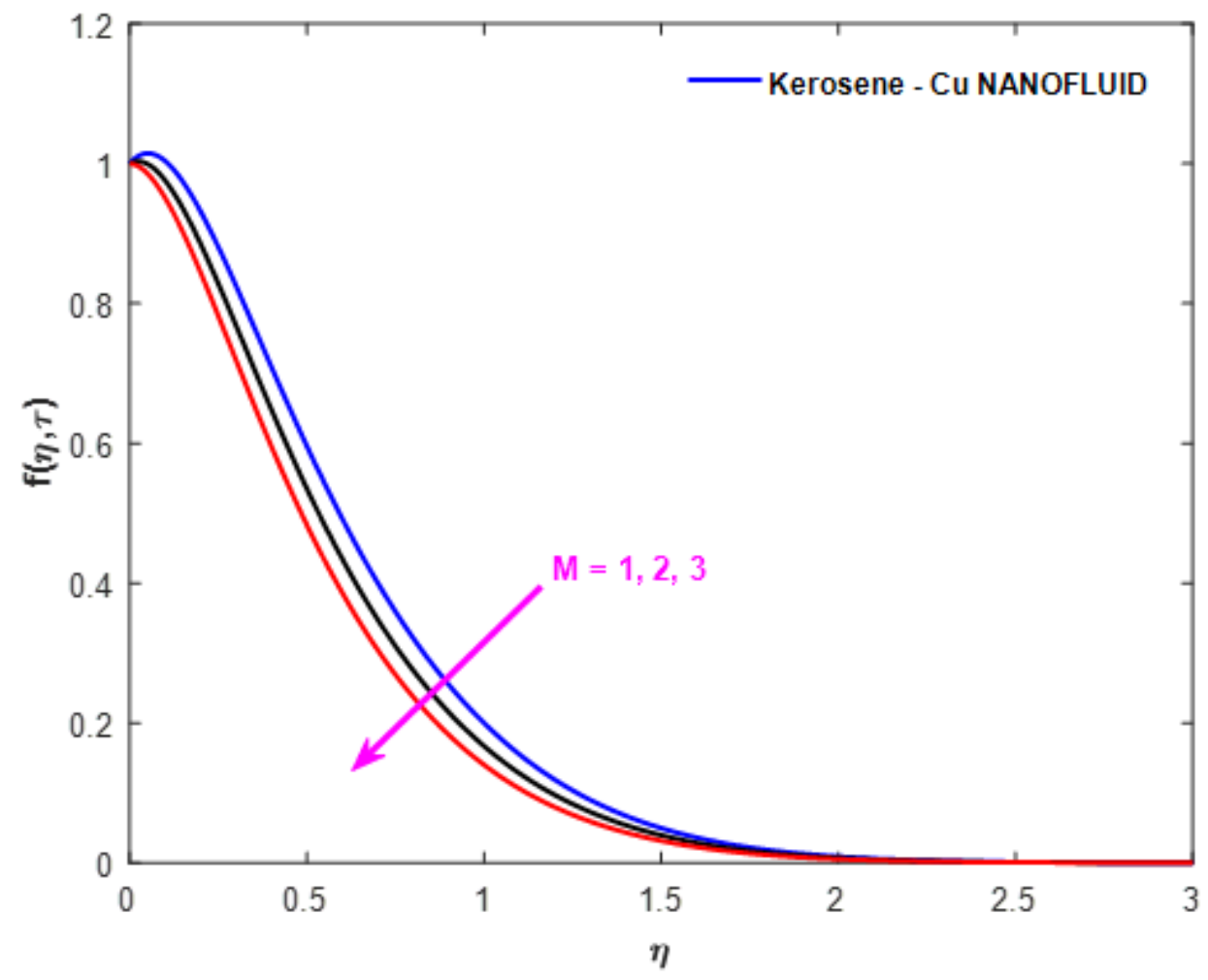

Figure 3. Velocity profile for the impact of $M$.

Figure 3 exhibits the performance of the magnetic strength on the fluid velocity profile due to $\mathrm{Cu}$-kerosene nanofluid. The insertion of the magnetic field experiences a force known as "Lorentz force" that causes a significant reduction in the velocity profile as well as the momentum thickness. A sharp fall in the profiles is rendered within the domain $\eta \leq 1$, and further is becomes smooth and ceases to zero.

Thermal buoyancy is the upthrust that causes a significant role in the velocity distribution, presented in Figure 4. 


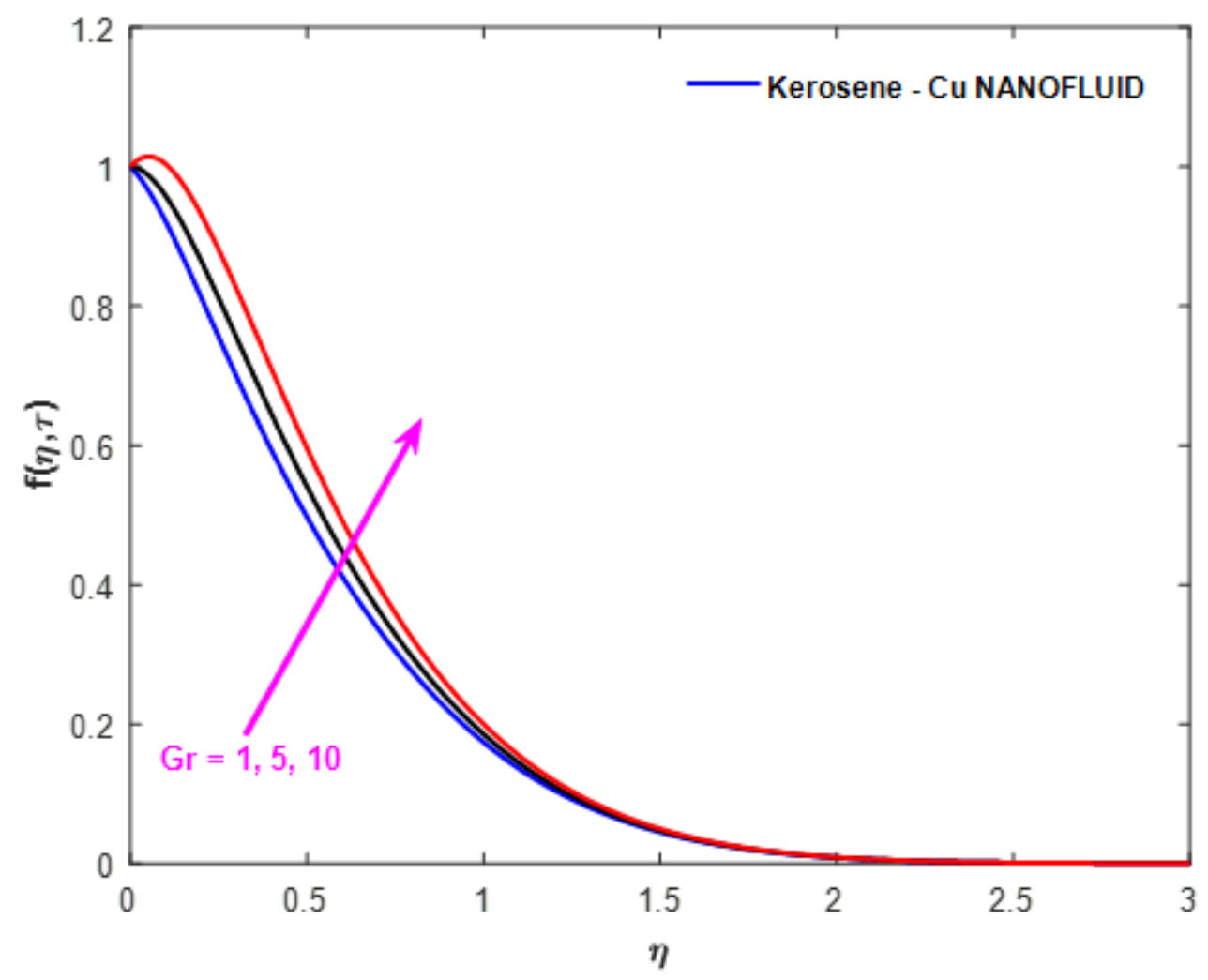

Figure 4. Velocity profile for the impact of $G r$.

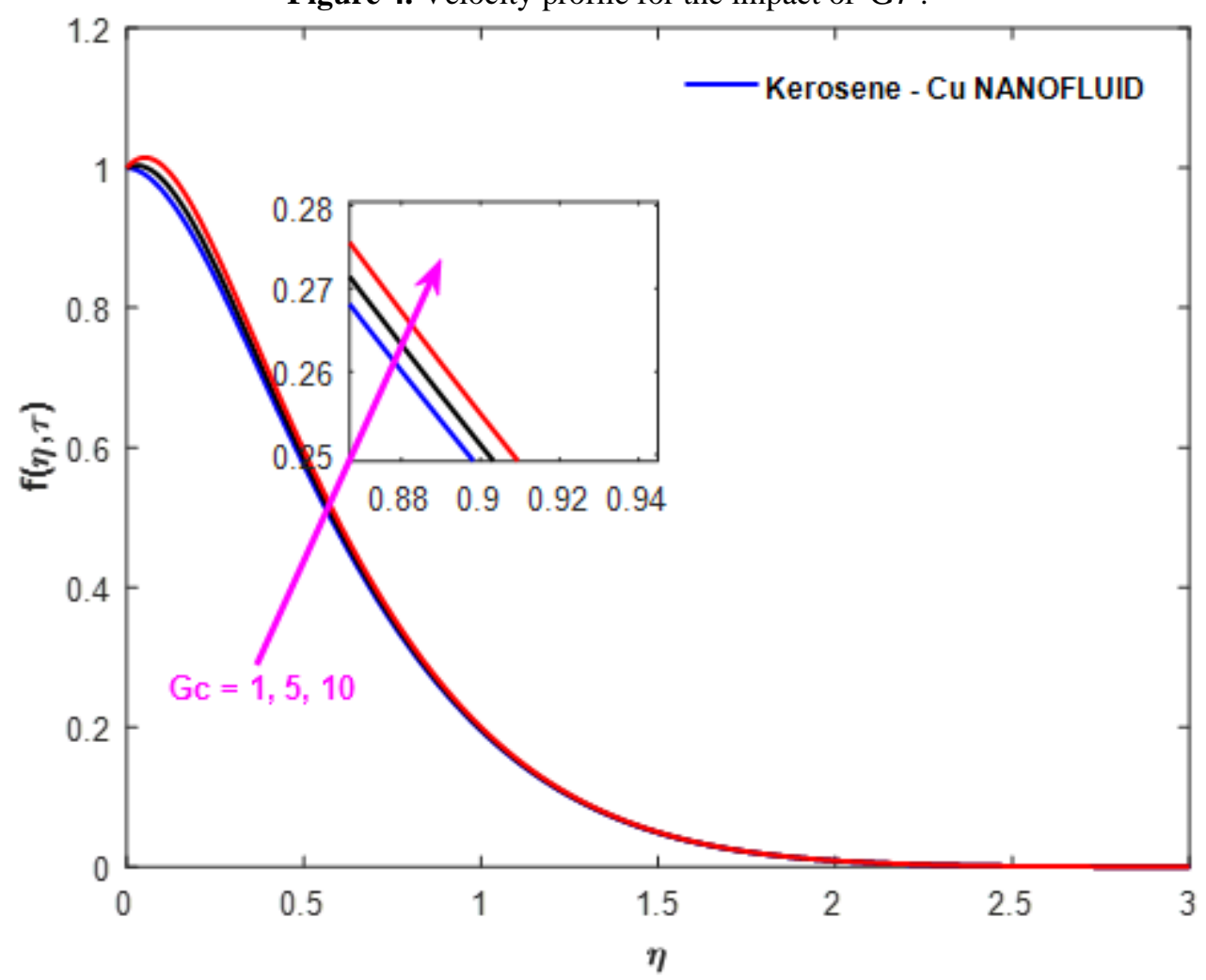

Figure 5. Velocity profile for the impact of $G c$.

Due to the interaction of buoyancy, the dense cold air has a lift to move the hot air up. This lift performs a major augmentation in the velocity, and the fact depends upon the size of the temperature difference between the hot and cold air. Here, $G r>0$ signifies the cooling of the plate, and also with greater cooling, the movement of the hot air enriches that overshoot the $\mathrm{Cu}$-kerosene velocity. Figure 5 describes the experience of the solutal buoyancy on the velocity profile of $\mathrm{Cu}$-kerosene nanofluid. The hike in the value of solutal buoyancy is due to the greater 
concentration difference between the wall and the ambient state. Further, an increase $G c$ encourages the velocity distribution. The unsteady free convective nanofluid flow phenomenon based upon time-dependent and the behavior of time variation is displayed in Figure 6.

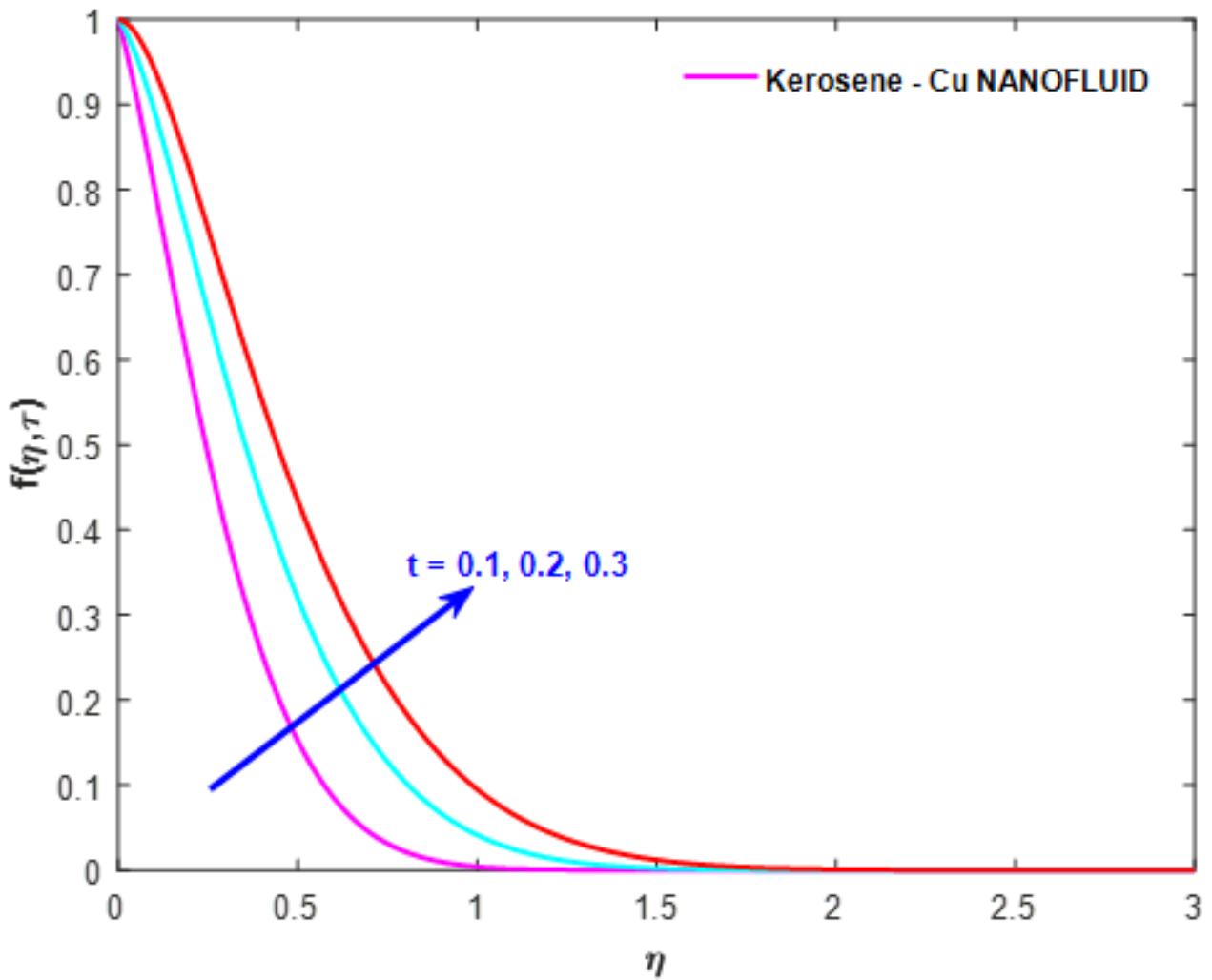

Figure 6. Velocity profile for the impact of $\boldsymbol{t}$.

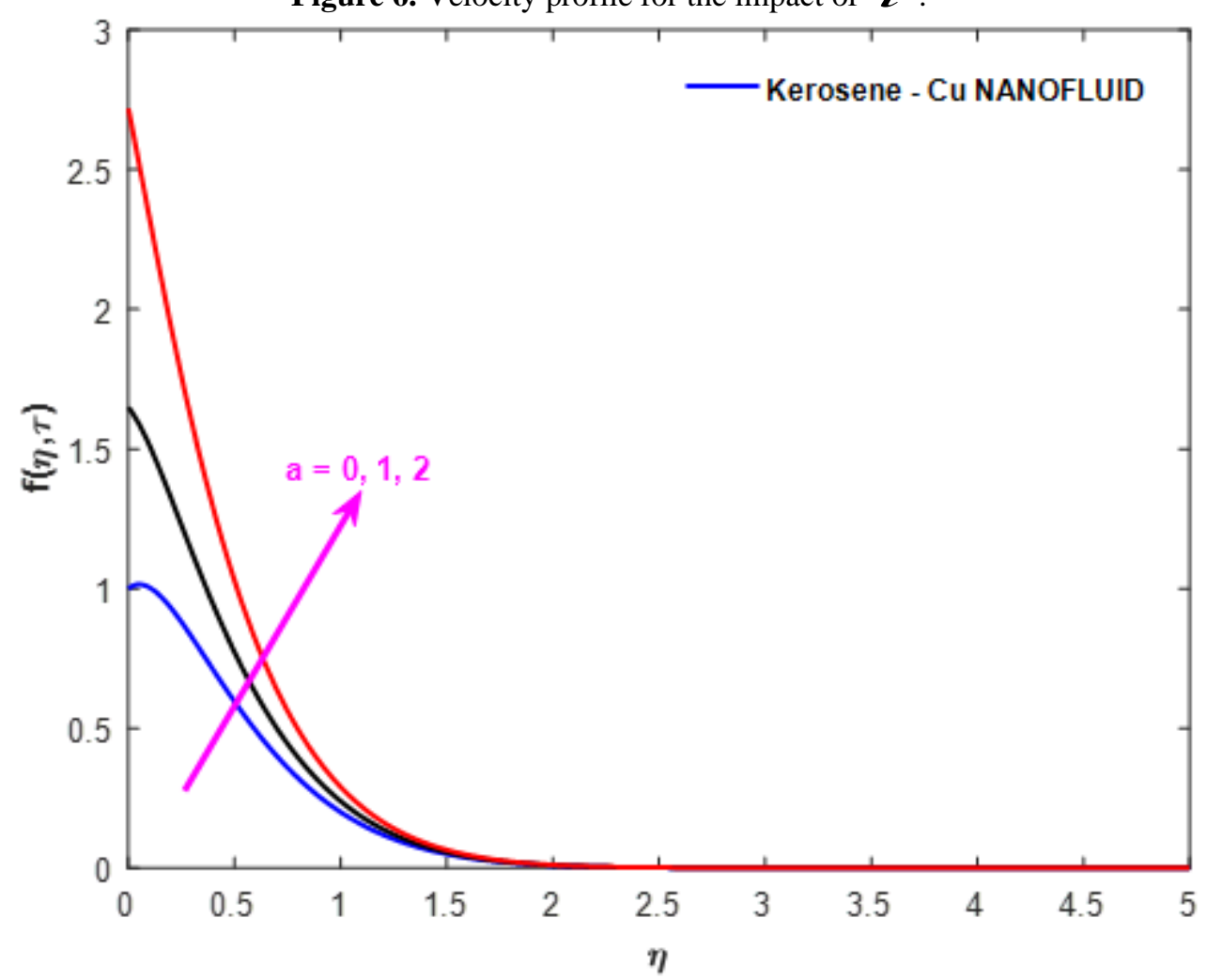

Figure 7. Velocity profile for the impact of $a$.

The profile explores with increasing period. It is very clear from the mathematical formulation of the problem. The formulation shows that the plate velocity has also played a vital role in nanofluids' velocity distribution, displayed in Figure 7 . With an increasing 
exponent parameter, the plate velocity also grows exponentially. Further, the enhanced value of an exponent of the fluid velocity enriches the profile within the entire flow domain.

\subsection{Temperature distributions.}

The variation of contributing parameters on the temperature of kerosene-based nanofluid is presented in this section. Figure 8 gathered important information about the particle concentration on the fluid temperature. Earlier literature confirms that $\mathrm{Cu}$ is a good conductor of heat. Increasing volume fraction leads to the combined effect of the base fluid with copper nanoparticles. The heavier density of the particle that retards the fluid velocity overshoots the fluid temperature within the entire domain.

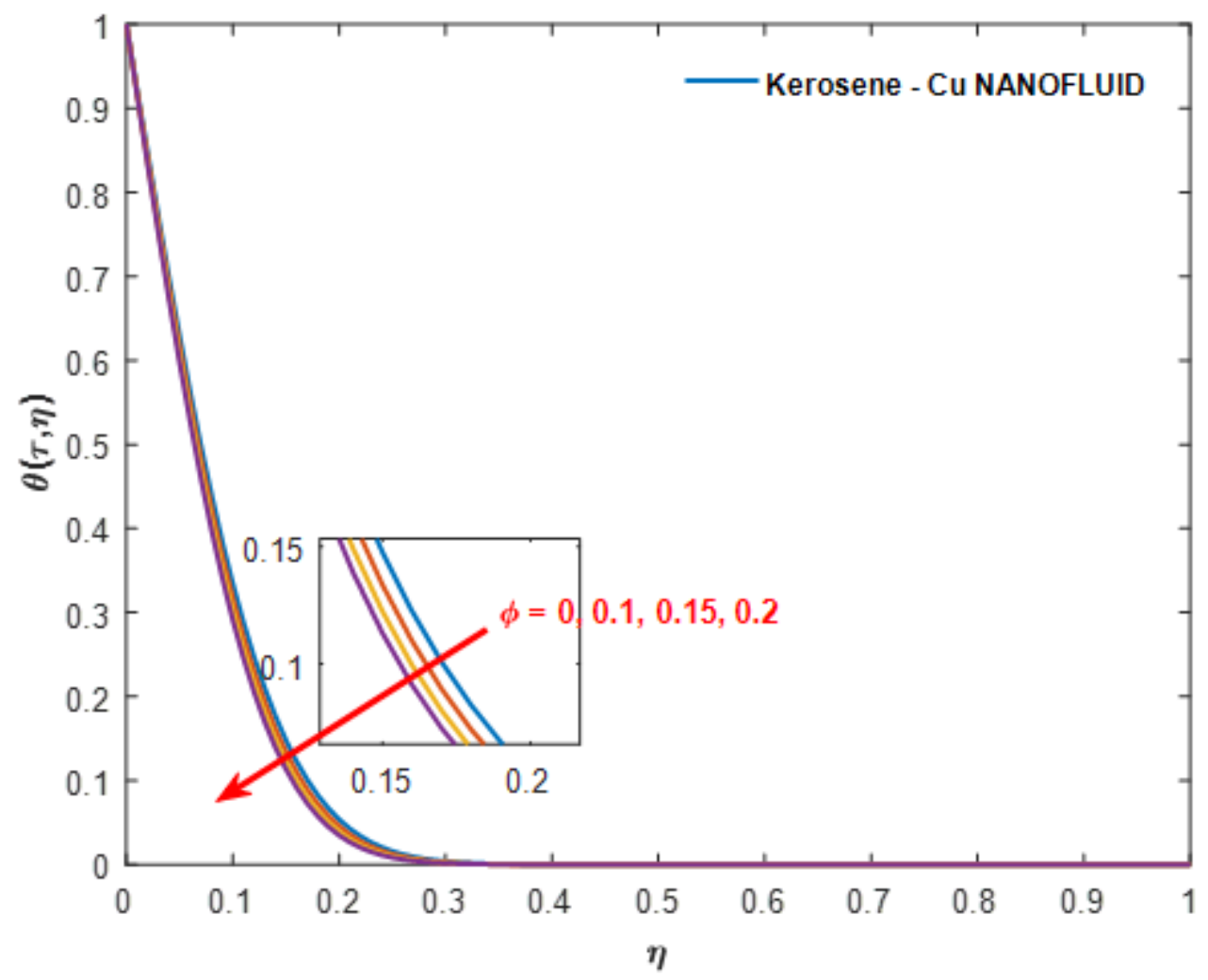

Figure 8. Temperature profile for the impact of $\phi$.

Figure 9 displays the characteristics of the Prandtl number on the fluid temperature. Pr is the ratio connecting to the kinematic viscosity and the thermal diffusivity. An augmentation in Pr clarifies that the retardation in the thermal diffusion and leads to decelerating the fluid temperature.

The profiles of fluid temperature are affected due to the interaction of thermal radiation and are exhibited in Figure 10. The transformation of radiation energy to electromagnetic radiation is proposed by thermal radiation. The release of electromagnetic waves from the fluid element is known to be radiative heat energy. The resulting figure shows a significant enhancement in the fluid temperature due to thermal radiation. 


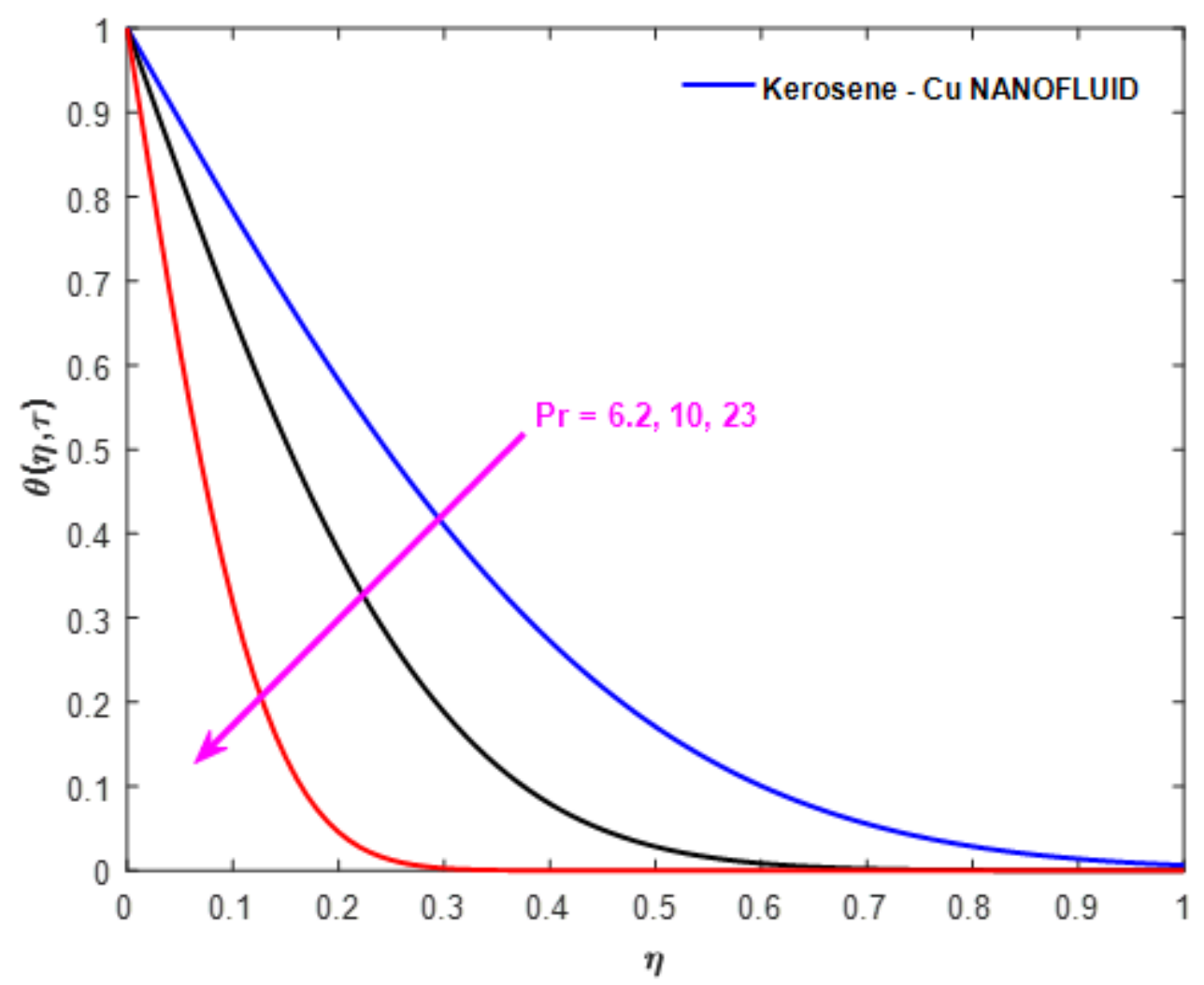

Figure 9. Temperature profile for the impact of $\operatorname{Pr}$.

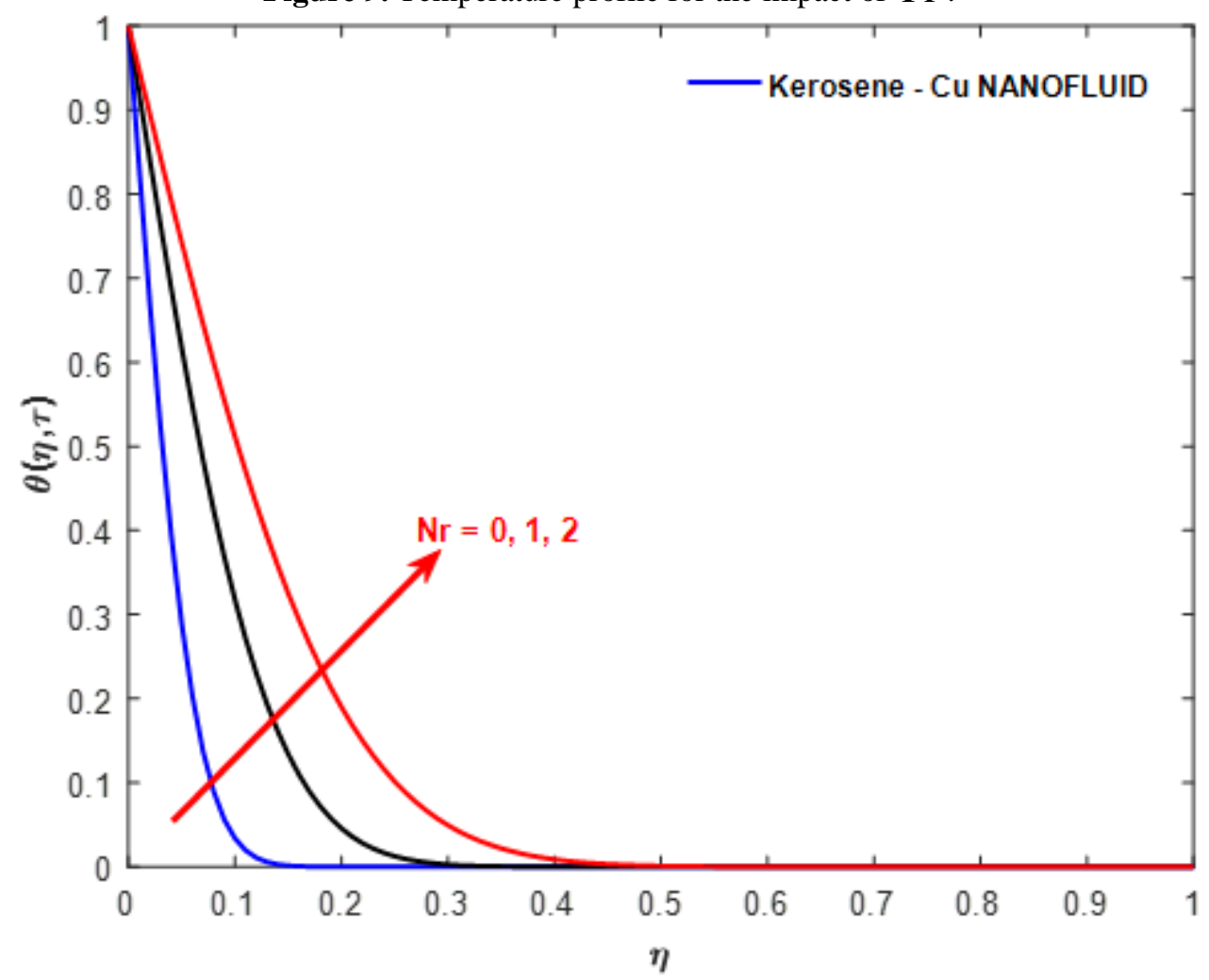

Figure 10. Temperature profile for the impact of $N r$. 


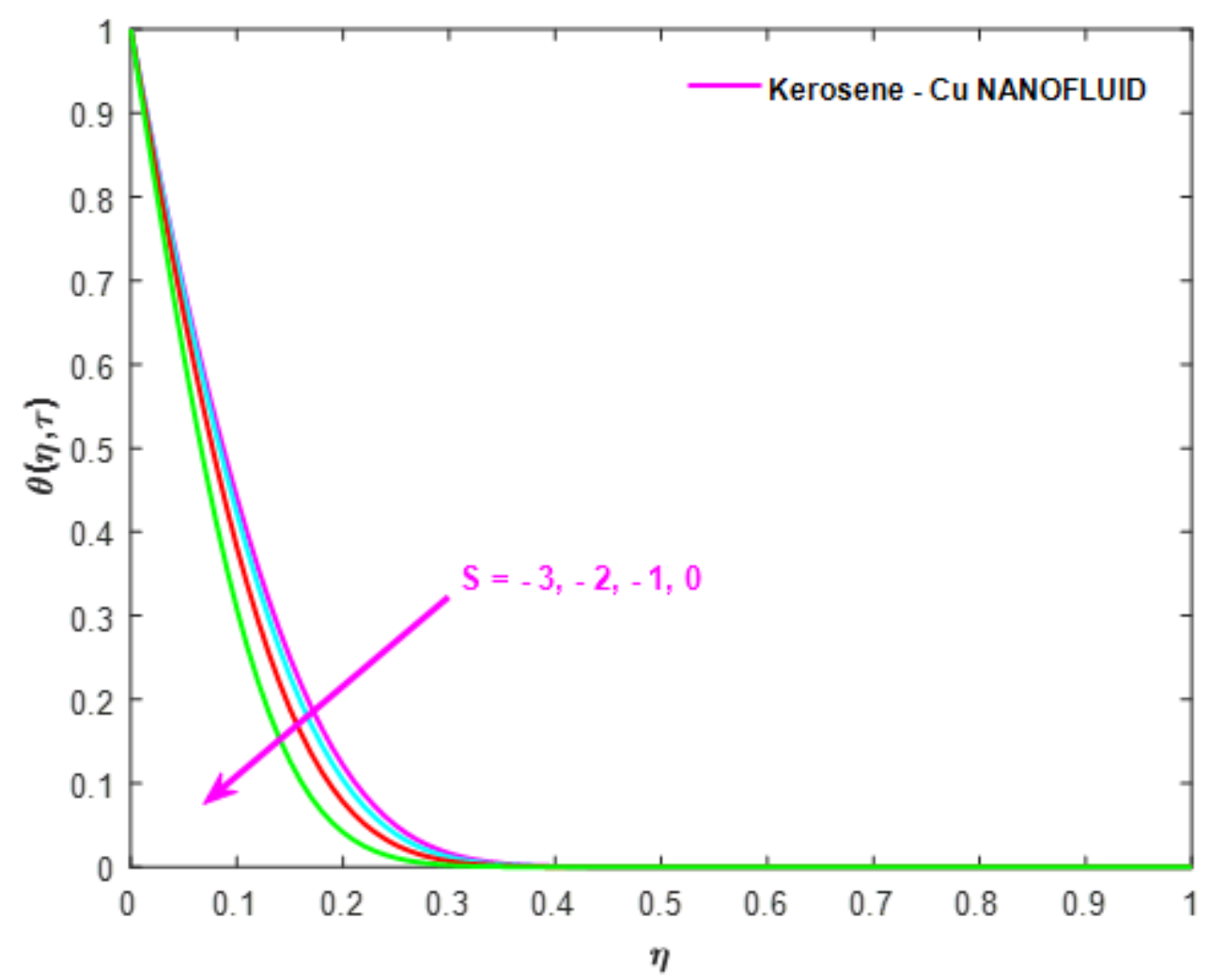

Figure 11. Temperature profile for the impact of $S$.

Figure 11 exhibits the impact of the heat sink on the fluid temperature. It is seen that an increasing heat sink retards the fluid temperature. Also, significant growth in the temperature is noticed for the enhanced values of the tie period shown in Figure 12.

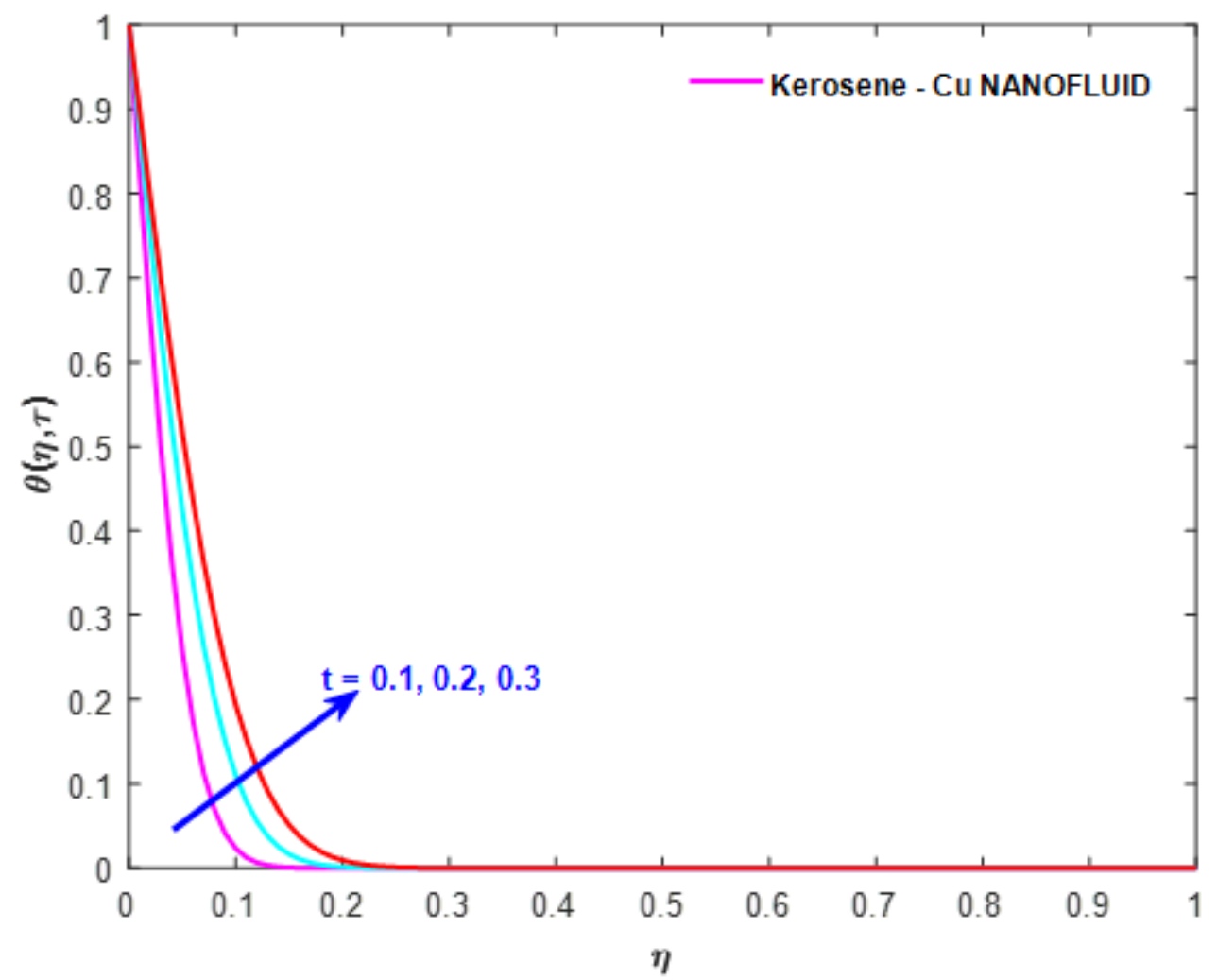

Figure 12. Temperature profile for the impact of $t$. 


\subsection{Concentration distribution.}

Considering the fluid concentration in the flow of $\mathrm{Cu}$-kerosene nanofluid is exhibited if the particle concentration is not completely agglomerated. Therefore, the existence of several parameters characterizes the fluid concentration.

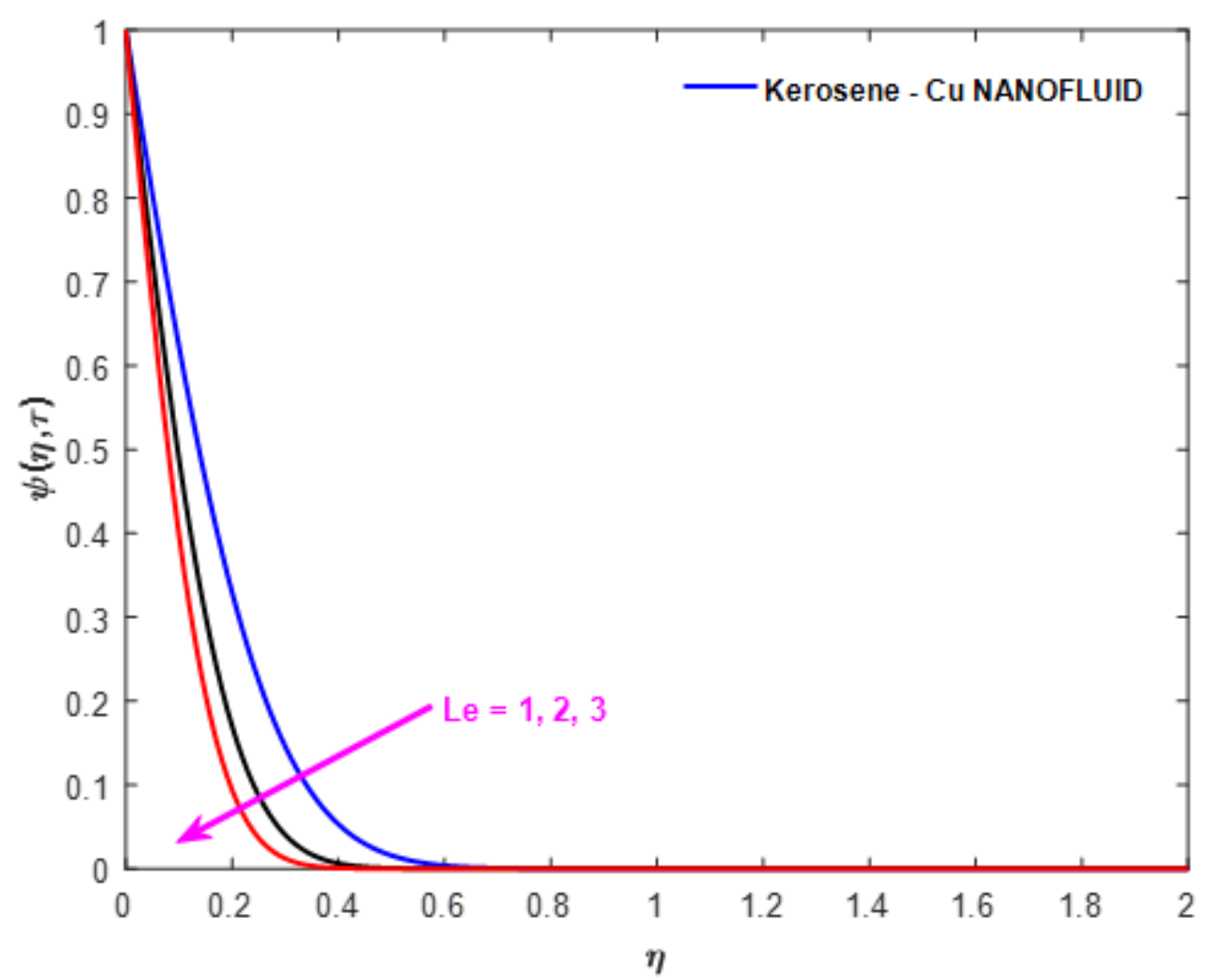

Figure 13. Concentration profile for the impact of $L e$.

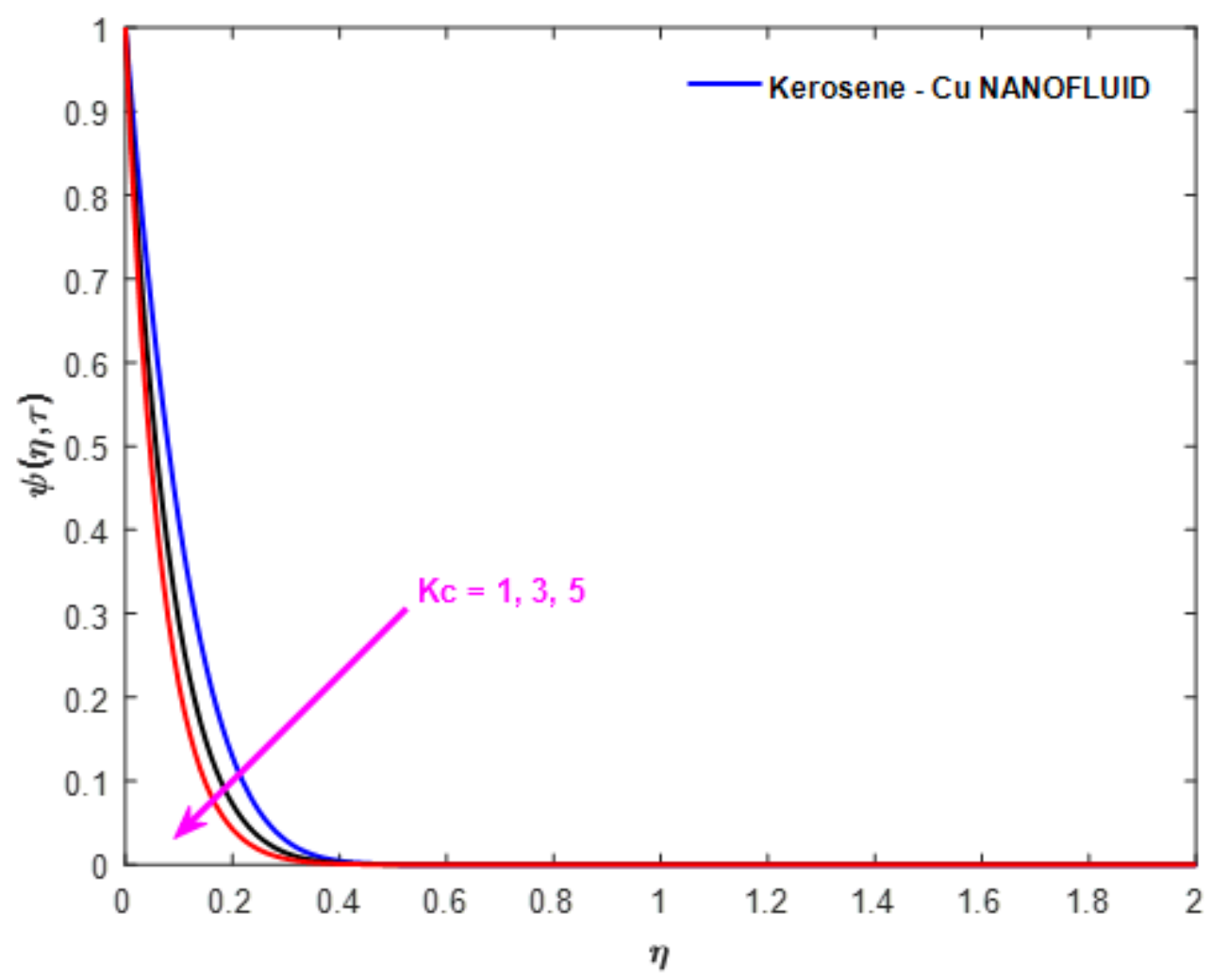

Figure 14. Concentration profile for the impact of $K c$. 
Figure 13 explores the behavior of the Lewis number on the fluid concentration profiles. Lewis number is expressed as the ratio of thermal and Brownian diffusivity. The augmentation in Le signifies the retardation in the Brownian diffusivity that resulted in the concentration profile retards in the entire domain. The interaction of several chemical species reactions on the concentration profile is displayed in Figure 14. Significant retardation is exhibited for the increasing chemical reaction. However, Figure 15 has a reverse trend for the enhanced period, i.e., an increase in period enhances the fluid concentration.

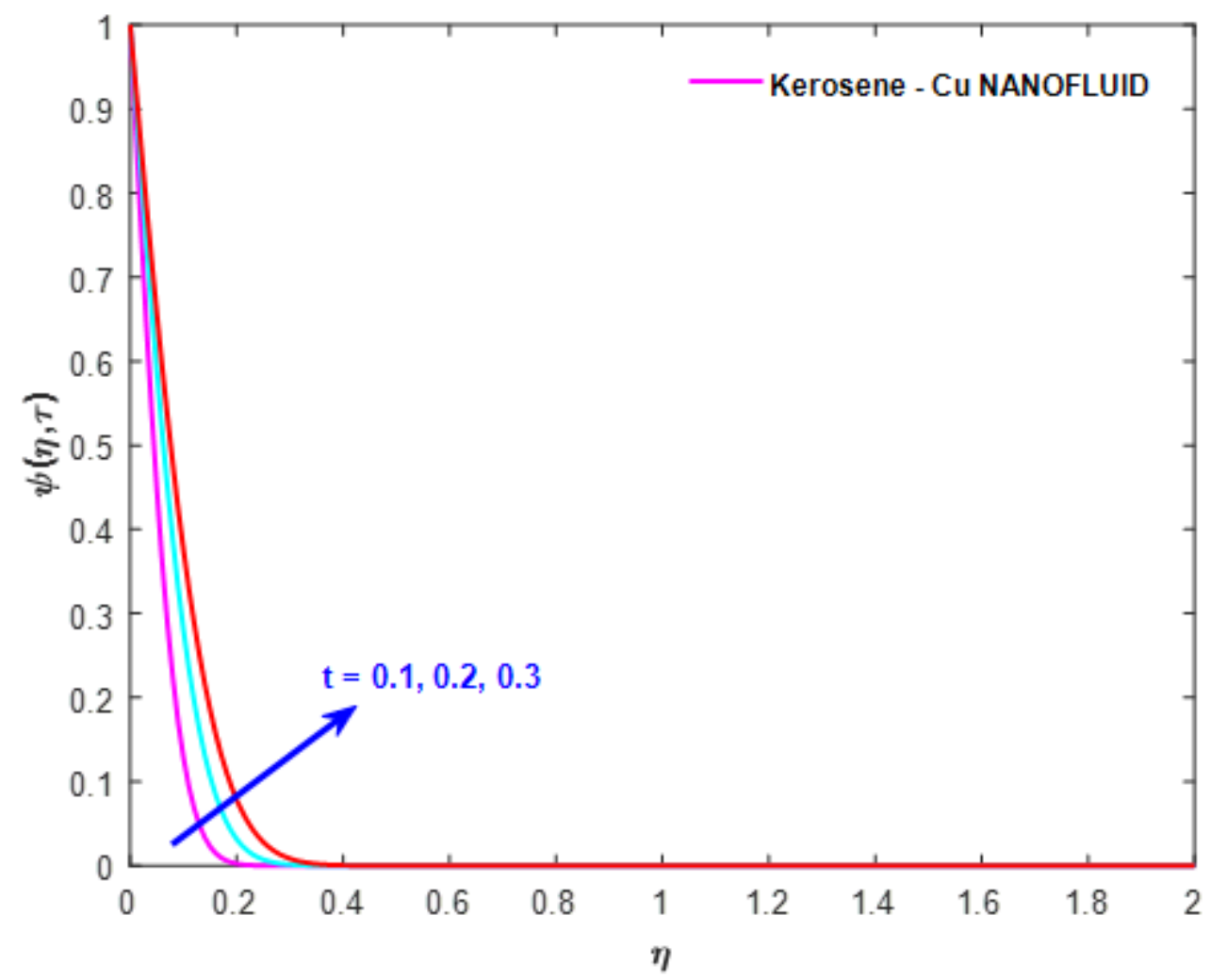

Figure 15. Concentration profile for the impact of $t$.

\subsection{Rate coefficients.}

The simulation of the several contribution parameters on the rate coefficients near the plate surface is obtained, and the numerical results are presented in Table 2.

Table 2. Numerical Simulation of Rate coefficients.

\begin{tabular}{c|c|c|c|c|c|c|c|c|c|c|c}
$\phi$ & $M$ & $G r$ & $G c$ & $\operatorname{Pr}$ & $N r$ & $S$ & $L e$ & $K c$ & $S k_{f}$ & $N u$ & $S h$ \\
\hline 0.1 & 1 & 10 & 10 & 23 & 1 & -0.1 & 2 & 0.1 & -0.7517 & 20.042 & 5.6798 \\
\hline 0.2 & & & & & & & & & 0.0822 & 22.0546 & \\
\hline 0.1 & 2 & & & & & & & & -0.3378 & 20.042 & \\
\hline & 3 & & & & & & & & 0.0399 & 20.042 & \\
\hline & 1 & 2 & & & & & & & 0.4931 & & \\
\hline & & 5 & & & & & & & 0.0263 & & \\
\hline & & 10 & 2 & & & & & & -0.0596 & & \\
\hline & & & 5 & & & & & & -0.3192 & & \\
\hline & & & 10 & 6.2 & & & & & -2.1079 & 5.544 & 2.9489 \\
\hline & & & & 23 & 2 & & & & -0.9918 & 20.1767 & 5.6798 \\
\hline & & & & & 3 & & & & -1.1692 & 20.2894 &
\end{tabular}




\begin{tabular}{c|c|c|c|c|c|c|c|c|c|c|c}
$\phi$ & $M$ & $G r$ & $G c$ & $\operatorname{Pr}$ & $N r$ & $S$ & $L e$ & $K c$ & $S k_{f}$ & $N u$ & $S h$ \\
\hline & & & & & 1 & -0.3 & & & -0.7196 & 19.4479 & \\
\hline & & & & & & -0.2 & & & -0.7355 & 19.7341 & \\
\hline & & & & & & -0.1 & 1 & & -1.0294 & 20.042 & 4.0163 \\
\hline & & & & & & & 3 & & -0.6157 & & 6.9564 \\
\hline & & & & & & 2 & 0.2 & -0.7399 & & 5.9438 \\
\hline & & & & & & & 0.3 & -0.7284 & & 6.2035
\end{tabular}

The shear rate coefficient at the plate encourages the profile with an increase in nanoparticle volume fraction and the magnetic parameter, whereas the retardation occurs due to the increase in the buoyant forces, i.e., increasing thermal and solutal buoyancy parameters. The rate of heat transfer increases with an augmentation in the Prandtl number, whereas the impact of thermal radiation opposes it significantly. Further, the Prandtl number also encourages the rate of solutal transfer. A favorable enhancement in the heat transfer rate is presented for the increasing values of the heat sink, and the rate of solutal transfer also favors the enhanced Lewis number and the chemical reaction parameter.

\section{Conclusions}

An analytical approach for the free convective flow of kerosene-based nanofluid past a moving vertical plate is presented in the current investigation. The impact of thermal radiation along with sink for the inclusion of the Mintsa model thermal conductivity enhances the thermal properties. The significance of diversified parameters is presented and elaborated clearly with their physical significance. Further, the conclusive remarks are displayed below as: The comparative result with an earlier investigation conforms to the convergence criteria of the analytical method employed herewith further it is one of the suggestive measures to carry forward the extensive exploration of the current proposal; The greater density of the nanoparticle and the resistance offered by the inclusion of the magnetic parameter tend to retards the fluid velocity significantly further, the buoyant forces, i.e., the thermal and solutal buoyancy encourages the profile; Fluid temperature hikes throughout the domain with enhanced volume fraction since the transport of heat through $\mathrm{Cu}$ is greater in comparison to the other nanoparticles; The transformation of the thermal radiation into the electromagnetic waves favors enhancing the nanofluid temperature; The fluid concentration decelerates for the significant growth in the Lewis number as well as the reactive agent; The skin friction coefficient hikes with suitable growth in the volume fraction, and the magnetic parameter further decelerate with increasing buoyant forces.

\section{Funding}

This research has no external fundings.

\section{Acknowledgments}

The manuscript has no acknowledgments.

\section{Conflicts of Interest}

The authors have no conflict of interest. 


\section{References}

1. Gurivi, P.R.; Raju, M.C., Mamatha, B., Varma, S.V.K. Thermal diffusion effect on MHD heat and mass transfer flow past a semi-infinite moving vertical porous plate with heat generation and chemical reaction. Applied Mathematics 2016, 7, 638-649, https://doi.org/10.4236/am.2016.77059.

2. Chambre, P.L.; Young, J.D. On the diffusion of a chemically reactive species in a laminar boundary layer flow. Physics of Fluids 1958, 1, 48-54, https://doi.org/10.1063/1.1724336.

3. Chen, T.S.; Yuh, C. F. Combined heat and mass transfer in natural convection along with the vertical cylinder. Int. J. Heat Mass Transfer 1980, 23, 451-461, https://doi.org/10.1007/BF01637127.

4. Patil, P.M.; Kulkarni, P.S. Effects of the chemical reaction on free convective flow of a polar fluid through a porous medium in the presence of internal heat generation. International Journal of theral Sciences. 2008, 47, 1043-1054, https://doi.org/10.1016/j.ijthermalsci.2007.07.013.

5. Tripathy, R.S.; Dash, G.C.; Mishra, S.R.; Baag, S. Chemical reaction effect on MHD free convective surface over a moving vertical plane through porous medium. Alexandria Engineering Journal 2015, 54, https://doi.org/10.1016/j.aej.2015.04.012.

6. Rout, P.K.; Sahoo, S.N.; Dash, G.C.; Mishra, S.R.. Chemical reaction effect on MHD free convection flow in a micropolar fluid. Alexandria Engineering Journal 2016, 55, 2967-73, https://doi.org/10.1016/j.aej.2016.04.033.

7. Jena, S.; Mishra, S.R.; Dash, G.C. Chemical reaction effect on MHD Jeffery fluid flow over a stretching sheet through porous media with heat generation/ absorption, Int. J. Appl. Comput. Math 2016, 25, 1-14. https://doi.org/10.1007/s40819-016-0173-8.

8. Misra, J.C.; Adhikary, S.D. MHD oscillatory channel flow, heat, and mass transfer in a physiological fluid in presence of chemical reaction. Alexandria Engineering Journal 2016, 55, 287-297, https://doi.org/10.1016/j.aej.2015.10.005.

9. Tripathy, R.S.; Dash, G.C.; Mishra, S.R.; Baag, S.. Chemical reaction effect on MHD free convective surface over a moving vertical plate through a porous medium. Alexandria Engineering Journal 2015, 54, 673-679, https://doi.org/10.1016/j.aej.2015.04.012.

10. Ahmed, S.; Kalita, K.; Chamkha, A. J. Analytical and numerical solution of three-dimensional channel flow in presence of sinusoidal fluid injection and a chemical reaction. Ain Shams Engineering Journal 2015, 6, 691-701, https://doi.org/10.1016/j.asej.2014.11.013.

11. Soundalgekar, V.M.. Viscous dissipation effects on unsteady free convective flow past an infinite vertical porous plate with constant suction. Int. J. Heat Mass Transfer 1972, 15, $1253 \quad$ - 61, https://doi.org/10.1016/0017-9310(72)90189-5.

12. Makinde, O.D.; Mishra, S. R. On stagnation point flow of variable viscosity nanofluids past a stretching surface with radiative heat. International Journal of Applied and Computational Mathematics 2017, 3, 561578, https://doi.org/10.1007/s40819-015-0111-1.

13. Eckert, E.R.G.; Drake, R.M. Heat and mass transfer, 2nd ed., Tata McGraw-Hill: New Delhi, India, 1979.

14. Hossain, A.M.; Alim, M.A.; Rees, D.A.S. The Effect of radiation on free convection from a porous a vertical plate. Int. J. Heat Mass Transfer 1999, 42, 181-191, https://doi.org/10.1016/S0017-9310(98)00097-0.

15. Makinde, O.D. Free convection flow with thermal radiation and mass transfer past a moving vertical porous plate. Int Commun. Heat Mass Transfer 2005, 32, 1411-19, https://doi.org/10.1016/j.icheatmasstransfer.2005.07.005.

16. Rohana, A.H.; Norihan, M.; Rosalind, N. Effects of radiation, Joule heating and viscous dissipation on MHD Marangoni convection over a flat surface with suction and injection. World Applied Sciences Journal 2013, 21, 933-938, https://doi.org/10.5829/IDOSI.WASJ.2013.21.6.2914.

17. Babu, P. R.; Rao, J. A.; Sheri, S. Radiation effect on MHD heat and mass transfer flow over a shrinking sheet with mass suction. Journal of Applied Fluid Mechanics 2014, 7, 641-650, https://doi.org/10.36884/jafm.7.04.21389.

18. Makinde, O.D.; Mishra, S.R. Chemically reacting MHD flow of variable viscosity fluid past a convectively heated vertical plate embedded in a porous medium. Defect and Diffusion Forum 2017, 374, 83-91, https://doi.org/10.4028/www.scientific.net/DDF.374.83.

19. Mishra, S.R.; Baag, S.; Bhatti, M. M. Study on Heat and Mass Transfer MHD Walters $B^{\prime}$ Nanofluid flow Induced by a Stretching Porous Surface. Alexandria Engineering Journal 2018, 57, 2445-53, https://doi.org/10.1016/j.aej.2017.08.007. 
20. Kayalvizhi, M.; Kalaivanan, R.; Ganesh, N.V. Velocity slip effects on heat and mass fluxes of MHD viscousOhmic dissipative flow over a stretching sheet with thermal radiation. Ain Shams Eng. 2016, 7, 791-797, https://doi.org/10.1016/j.asej.2015.05.010.

21. Rashidi, M.M.; Sheremet, M.A.; Sadri, M.; Mishra, S.R.; Pattnaik, P.K.; Rabiei, F.; Abbasbandy, S.; Sahihi, H.; Erfani, E. Semi-Analytical Solution of Two-Dimensional Viscous Flow through Expanding/Contracting Gaps with Permeable Walls. Math., Comput. Appl. 2021, 26, 41, https://doi.org/10.3390/mca26020041.

22. Abbasi, F.M.; Shehzad, S.A.; Hayat, T.; Alsaedi, A.; Obid, M. A. Influence of heat and mass flux conditions in the hydromagnetic flow of Jeffrey nanofluid. AIP ADVANCES 2015, 5, 1-13, https://doi.org/10.1063/1.4914549.

23. Geetha, E.; Muthucumaraswamy, R. Free convective heat and mass transfer induced by a constant mass flux on a parabolic started vertical plate with variable temperature. International Journal of Advanced Scientific Technologies in Engineering and Management Sciences 2018, 2, 1-12, https://doi.org/10.13140/RG.2.2.29789.05600.

24. Hayat, T.; Ullah, I.; Muhammad, T.; Alsaedi, A.; Shehzad, S. A. Three-dimensional flow of Powell-Eyring nanofluid with heat and mass flux boundary conditions. Chin. Phys. B. 2016, 25, 1-8, https://doi.org/10.1088/1674-1056/25/7/074701.

25. Mishra, A.K.; Pattnaik, P.K.; Mishra, S.R.; Senapati, N. Dissipative heat energy on $\mathrm{Cu}_{\text {and }} \mathrm{Al}_{2} \mathrm{O}_{3}$ ethyleneglycol-based nanofluid flow over a heated semi-infinite vertical plate. Journal of Thermal Analysis and Calorimetry 2020, 145, https://doi.org/10.1007/s10973-020-09666-z.

26. Jena, S.; Mishra, S. R.; Pattnaik, P.K.; Sharma, R.P. Nanofluid flow between parallel plates and heat transfer in presence of chemical reaction and porous matrix. Latin American Applied Research 2020, 50, 283-289, https://doi.org/10.52292/j.laar.2020.476.

27. Parida, S.K.; Mishra, S.R.; Dash, R.K.; Pattnaik, P.K.; Chu; Y.-M.; Shah, F. Dynamics of dust particles in a conducting water based kerosene nanomaterials: A computational approach. International Journal of Chemical Reactor Engineering 2021, https://doi.org/10.1515/ijcre-2020-0204.

28. Rout, B.C.; Mishra, S.R. Thermal energy transport on MHD nanofluid flow over a stretching surface: A comparative study. Engineering Science and Technology, an International Journal 2018, 21, 60-69, https://doi.org/10.1016/j.jestch.2018.02.007.

29. Pattnaik, P.K.; Biswal, T. Analytical solution of MHD free convective flow through porous media with time dependent temperature and concentration. Walailak J. of Sci \& Tech 2015, 12, 749-762, https://doi.org/10.14456/WJST.2015.57.

30. Mishra, S.R.; Pattnaik, P.K.; Bhatti, M.M.; Abbas, T. Analysis of heat and mass transfer with MHD and chemical reaction effects on viscoelastic fluid over a stretching sheet. Indian Journal of Physics 2017, 91, 1219-27, https://doi.org/10.1007/s12648-017-1022-2.

31. Pattnaik, P.K.; Mishra, S.R.; Barik, A.K.; Mishra, A.K.; Influence of chemical reaction on magnetohydrodynamic flow over an exponential stretching sheet: a numerical study. International Journal of Fluid Mechanics Research 2020, 47, 1-12, https://doi.org/10.1615/InterJFluidMechRes.2020028543.

32. Pattnaik, P.K.; Jena, S.; Dei, A.; Sahu, G. Impact of chemical reaction on micropolar fluid past a stretching sheet. JP Journal of Heat and Mass Transfer 2019, 18, 207-223, https://doi.org/10.17654/HM018010207.

33. Pattnaik, P.K.; Mishra, S.R.; Mahanthesh, B.; Gireesha, B.J.; Gorji, M.R. Heat transport of nano-Micropolar fluid with an exponential heat source on a convectively heated elongated plate using Numerical computation. Multidiscipline Modeling in Materials and Structures 2020, 16, 1295-1312, https://doi.org/10.1108/MMMS12-2018-0222.

34. Barik, A.K.; Mishra, S.K.; Mishra, S.R.; Pattnaik, P.K. Multiple Slip effects on MHD nanofluid flow over an inclined, radiative and chemically reacting stretching sheet by means of FDM. Heat transfer Asian research 2020, 49, 477-501, https://doi.org/10.1002/htj.21622.

35. Mishra, S.R.; Pattnaik, P.K.; Dash, G.C. Effect of heat source and double stratification on MHD free convection in a micropolar fluid. Alexandria Engineering Journal 2015, 54, 681-689, https://doi.org/10.1016/j.aej.2015.04.010.

36. Mishra, S.R.; Mahanthesh, B.; Mackolil, J.; Pattnaik, P.K. Nonlinear radiation and cross-diffusion effects on the micropolar nanoliquid flow past a stretching sheet with an exponential heat source. Heat transfer Asian research 2021, 50, 3530-46, https://doi.org/10.1002/htj.22039.

37. Ali, B.; Pattnaik, P.K.; Naqvi, R.A.; Waqas, H.; Hussain, S. Brownian motion and thermophoresis effects on bioconvection of rotating Maxwell nanofluid over a Riga plate with Arrhenius activation energy and 
Cattaneo-Christov heat flux theory. Thermal Science and Engineering Progress 2021, 23, 100863, https://doi.org/10.1016/j.tsep.2021.100863.

38. Pattnaik, P.K.; Mohapatra, D.K.; Mishra, S.R. Influence of Velocity Slip on the MHD Flow of a Micropolar Fluid over a Stretching Surface. Lecture Notes in Mechanical Engineering 2021, 307-321, https://doi.org/10.1007/978-981-15-9817-3_21.

39. Pattnaik, P.K.; Pattnaik, J.R.; Mishra, S.R.; Nisar, K.S. Variation of the shape of $\mathrm{Fe}_{3} \mathrm{O}_{4}$-nanoparticles on the heat transfer phenomenon with the inclusion of thermal radiation. Journal of Thermal Analysis and Calorimetry 2021, https://doi.org/10.1007/s10973-021-10605-9.

40. Pattnaik, P.K.; Mishra, S.R.; Bhatti, M.M. Duan-rach approach to study $\mathrm{Al}_{2} \mathrm{O}_{3}$-ethylene glycol $\mathrm{C}_{2} \mathrm{H}_{6} \mathrm{O}_{2}$ nanofluid flow based upon KKL model. Inventions 2021, 5, 45, https://doi.org/10.3390/inventions.

41. Das, S.; Jana, R.N. Natural convective magneto-nanofluid flow and radiative heat transfer past a moving vertical plate. Alexandria Eng. J. 2015, 54, 55-64, https://doi.org/10.1016/j.aej.2015.01.001.

42. Kandelousi, M.S.; Ganji, D.D. External Magnetic Field Effects on Hydrothermal Treatment of Nanofluid, $1^{\text {st }}$ ed.; The Boulevard: Langford Lane, Kidlington, UK, Oxford, OX5 1GB, 222016.

43. Mintsa, A.H.; Roy, G.; Nguyen, C.T.; Doucet, D. New temperature dependent thermal conductivity data for $\begin{array}{lllllll}\text { water-based } \text { nanofluids. Int } J \quad \text { Therm } & \text { Sci. }\end{array}$ https://doi.org/10.1016/j.ijthermalsci.2008.03.009.

44. Sheikholeslami, M.; Ganji, D.D. Ferrofluid flow and heat transfer in a semi annulus enclosure in the presence of magnetic source considering thermal radiation. J. Taiwan Inst. Chem. Eng. 2015, 47, 6-17, https://doi.org/10.1016/j.aej.2016.11.007.

45. Mahanthesh, B.; Gireesha, B.J.; Gorla, R.S.R. Heat and mass transfer effects on the mixed convective flow of chemically reaction nanofluid past a moving/stationary vertical plate. Alexandria Engineering Journal 2016, 55, 569-581, https://doi.org/10.1016/j.aej.2016.01.022. 